\title{
"Les ONG internationales dans le tourbillon du développement. Quelques réflexions à partir de la situation au Laos
}

François Guégan

\section{(2) OpenEdition}

Journals

\section{Édition électronique}

URL : https://journals.openedition.org/conflits/2005

DOI : $10.4000 /$ conflits. 2005

ISSN : $1777-5345$

Éditeur :

CECLS - Centre d'études sur les conflits - Liberté et sécurité, L'Harmattan

Édition imprimée

Date de publication : 1 décembre 2005

ISBN : 2-296-00230-7

ISSN : 1157-996X

\section{Référence électronique}

François Guégan, «"Les ONG internationales dans le tourbillon du développement. Quelques réflexions à partir de la situation au Laos », Cultures \& Conflits [En ligne], 60 | hiver 2005, mis en ligne le 10 mars 2006, consulté le 27 septembre 2021. URL : http://journals.openedition.org/conflits/2005 ; DOI : https://doi.org/10.4000/conflits.2005

Ce document a été généré automatiquement le 27 septembre 2021.

Creative Commons License 


\title{
"Les ONG internationales dans le tourbillon du développement. Quelques réflexions à partir de la situation au Laos
}

\author{
François Guégan
}

1 Au Laos comme ailleurs, la Guerre froide est aujourd'hui terminée. La dissipation des stratégies des blocs a libéré les Laotiens d'un étau dont ils sont longtemps restés prisonniers. Divisé par les objectifs politiques et militaires des deux grands pendant plus de vingt ans, uni sous la gouverne communiste jusqu'à ce que Moscou s'effondre, le Laos reste aujourd'hui à la croisée des chemins. Entre dictature communiste et ouverture économique, ce sont les acteurs de l'aide au développement qui maintiennent le pays à flot. Les motivations de l'aide internationale ne sont aujourd'hui plus les mêmes, mais elles relèvent d'objectifs non moins discernables. Si la RDP Lao reçoit le soutien des institutions financières internationales, des Nations unies et d'une multitude de pays, elle doit désormais entreprendre des réformes qui satisfont ses bailleurs sans forcément s'appuyer sur les demandes des populations. La logique latente pourrait se résumer comme suit: «Faites ce que l'on vous dit, et nous vous aiderons ».

2 Ce que l'on demande aux dirigeants laotiens, c'est de libéraliser l'économie et de réduire les dépenses de l'Etat. Le tout pour «intégrer » l'économie régionale et internationale. Le respect des droits de l'Homme, néanmoins, ne leur est pas imposé avec la même ferveur. Et si les acteurs institutionnels misent de plus en plus, quoique timidement, sur la santé et l'éducation, l'objectif final reste, comme le dit clairement la coopération australienne au Laos, « d'avancer les intérêts [des pays bailleurs] en favorisant les conditions pour la réduction de la pauvreté et le développement durable ${ }^{1}$ ». Ces intérêts sont aujourd'hui principalement économiques, les pays du Nord ayant besoin des nouveaux marchés que constituent les pays en voie de développement. L'aide publique au développement est un investissement pour l'avenir. Elle est en même temps la 
poursuite de la politique extérieure et le moyen pour les pays riches de donner une image "généreuse " d'eux-mêmes sur la scène internationale². Les ONG, elles, sont souvent considérées comme échappant à ces logiques. Il est vrai que certains de leurs personnels accomplissent des missions longues, proches des populations, et parfois dans des endroits très reculés. Leur identité, leurs motivations et leurs moyens diffèrent, il est vrai, des bailleurs institutionnels. Leur histoire aussi, est différente. Comme l'a dit Bernard Kouchner lors de la création de Médecins du monde : «Qui nous a commis ? Personne. Et c'est cela qui fait notre droit ${ }^{3}$ ».

3 Mais la réalité sur le terrain ne répond pas toujours à ces attentes. Premièrement, l'apparence des acteurs de l'aide internationale, ONG ou non, est souvent la même. Tous sont issus de pays occidentaux développés (et du Japon). Quatre-vingt-douze des cent quatre ONG internationales recensées par le Programme des Nations unies pour le développement (PNUD) et les services de la coopération internationale au Laos entre 1986 et 2003 sont originaires de pays occidentaux. Huit sont japonaises, trois thaïlandaises, une seule coréenne. Les responsables des organisations de l'aide, à Vientiane ou dans les bureaux des projets, sont par conséquent quasiment tous d'apparence européenne. Il est peu probable que les Laotiens, à moins qu'ils soient impliqués dans les réseaux du développement, fassent la différence entre les multiples acteurs de l'aide. Ces derniers, dès que la taille de leur organisation ou leur salaire le leur permet, roulent dans d'imposants $4 \times 4$ rutilants. La fait qu'ils soient estampillés "Japon ", « ONU », "Save the Children », ou "Banque mondiale » a finalement assez peu d'importance. De loin, ce sont les mêmes. Les nuances existent, bien sûr, mais il faut avoir une certaine connaissance des systèmes en place pour pouvoir dire qui fait quoi, et selon quels principes. Les acteurs de l'aide internationale restent entre eux pour la plupart, se retrouvent dans les mêmes restaurants, les plus chers, inaccessibles pour la plupart des Laotiens, fussent-ils de temps en temps à leur contact. Au sein de cette communauté des "expats ", on partage bien souvent les mêmes centres d'intérêts, les mêmes salaires, le même pouvoir, et le même décalage obséquieux avec la réalité du pays. Les cas contraires sont assez rares pour faire figure d'exceptions confirmant la règle.

4 Plus en amont, les ressources humaines des ONG ont, elles aussi, tendance à se rapprocher de celles des institutions gouvernementales. Depuis la naissance du mouvement «sans frontières ", illustrée par les débuts de MSF en 1971 avec la crise du Biafra, les personnels des ONG se sont largement professionnalisés ${ }^{4}$. Dans les bureaux des grandes - et pas toujours si grandes - ONG, l'idéal d'humanité et d'entraide volontaire, s'il n'a pas disparu, a laissé une grande place aux diplômés des grandes écoles (Sciences Po, écoles de commerce, etc.). De plus en plus, les personnels de terrain, comme au siège, ont fait de nombreuses années d'études. Souligner poliment cette évolution consiste à dire que «l'engagement humanitaire n'est plus seulement militant, mais également professionnel $»^{5}$. Les jeunes qui arrivent aujourd'hui sur le terrain ont souvent plus de diplômes et de certitudes que d'expérience et de convictions. Dans les grandes ONG, on préfère embaucher un «chef » de mission familier des procédures de financement des bailleurs de fonds, plutôt qu'une personne connaissant le Laos, sa (ses) culture(s) et ses subtilités. Cette évolution est bénéfique par certains aspects, car les programmes y gagnent en "professionnalisme ». Mais les éléments de proximité, d'engagement volontaire, et d'investissement personnel en pâtissent. Les responsables de terrain et les directeurs d'ONG peuvent ainsi passer d'une ONG à l'autre puis d'une ONG à une institution gouvernementale nationale ou internationale et vice-versa. Les 
acteurs de terrain (administrateurs, logisticiens, techniciens, etc.) peuvent travailler un moment pour une ONG, un autre pour l'ONU, un autre pour la Banque mondiale, sans que l'éthique vienne s'interposer. Dans un pays où la plupart des gens vivent avec moins de deux dollars par jour, le salaire versé aux « expats » (qui descend rarement en dessous de 1000 dollars mensuels) est bien souvent le nerf de la guerre. Les ONG, si elles veulent garder un personnel expatrié de qualité, doivent s'aligner sur la concurrence. Les expatriés chômeurs, quant à eux, cherchent du travail où il y en a, où qu'il soit. Encore une fois, les exceptions existent, mais les idéalistes, de manière générale, ont peu de place sur le marché du développement.

5 Le fait que les grandes ONG vivent largement des fonds publics renforce le sentiment de confusion. En effet, le terme «non gouvernemental» sous-entend nécessairement de ne pas s'impliquer dans les mécanismes étatiques. D'où le succès récemment remporté par le terme «OSI» (Organisations de Solidarité Internationale). Pour les personnes non initiées au milieu du développement, ces subtilités restent du domaine du un flou artistique, car les croyances veulent que les ONG soient indépendantes des Etats. Mais à $\mathrm{y}$ regarder de plus près, il est facile de se perdre en considérations. Le gouvernement laotien, lui aussi, ne peut pas toujours faire la différence - et en est d'autant plus méfiant. Comment, objectivement, prétendre être «indépendant» lorsqu'on est financé à plus de $50 \%$ par le même bailleur institutionnel ? Ce dernier émet une influence, ne serait-ce que par la possible suspension de ses fonds, qui conduirait à l'arrêt du projet. En dehors de toutes les considérations techniques de terrain (achat de matériel, rédaction d'avenants, etc.), si une institution est bailleur majoritaire, c'est à elle que l'ONG doit d'abord rendre des comptes, pas aux Laotiens. La dépendance est encore plus importante pour les ONG travaillant sur fonds délégués des coopérations bilatérales. En étant simples opératrices sur des projets pensés par les bailleurs, elles sont partie intégrante du rouage gouvernemental.

6 D'autre part, si les moyens mis en œuvre diffèrent, les orientations fondamentales de développement avancées par les ONG ne sont pas différentes de celles revendiquées par les autres acteurs. Comme le souligne Rony Brauman, président de MSF France de 1982 à 1994, «à voir les pratiques de ceux qui se perçoivent comme des agents de développement, l'esprit de la 'mission civilisatrice' a survécu à la disparition de l'impérialisme colonial ${ }^{6}$. "Cette « mission » revêt toujours les mêmes fondements, qu'elle soit d'hier ou d'aujourd'hui : le « paradigme altruiste » et le "paradigme modernisateur », explicités par Jean-Pierre Olivier de Sardan ${ }^{7}$ et repris par Brauman. Le premier consiste à faire « le bien pour les autres $^{8}$ ", et le second à diffuser "le progrès technique et économique ${ }^{9}$ " Ces deux éléments sont présents dans la plupart des projets et programmes de développement, au Laos comme ailleurs : «modernisation » des échanges économiques et favorisation de la concurrence ; introduction de nouvelles méthodes de culture et augmentation du rendement agricole; constructions de barrages et augmentation de la production énergétique; ou encore programmes de santé primaire ou "d'éducation sanitaire »- consistant « prosaïquement à vanter les mérites du savon et des latrines (...) dans le domicile de familles où les [développeurs] n'ont pas été conviés ${ }^{10} »$.Tous ces programmes et projets ont pour but de faire avancer les populations vers le progrès, de la même manière que les colons, aussi humanistes qu'ils pussent être, légitimaient la colonisation parce qu'elle était "source de bienfaisance et d'élévation des mœurs, une obligation de conscience qui se déduisait de l'évidente supériorité de la société colonisatrice sur les peuplades concernées ${ }^{11} »$. En ce sens, les ONG ne sont qu'un élément, certes distinct, 
mais constituant, du même projet, économiquement et culturellement néocolonialiste (bien qu'il le soit parfois d'une façon inconsciente).

7 Le discours de certains acteurs de terrain vient confirmer ce constat général. Combien d'entre eux, qu'ils travaillent pour l'ONU, une ONG ou quelconque coopération bilatérale, se plaignent régulièrement, au premier ou second degré, de la "paresse " des Laotiens... Ils remplissent les bars de Vientiane sur le bord du Mékong, et discourent de leur dernière mission, leur dernier projet, qui a dû subir la lenteur de l'administration laotienne, le manque de motivation des personnels de santé pour les formations dispensées, les perpétuelles demandes d'augmentation de perdiem, etc. Ces considérations, si elles sont le reflet d'une certaine réalité, ne sont pas sans rappeler le discours darwiniste des colons français, exaspérés par la nonchalance des Laotiens : ces êtres « simples, naïfs, crédules, ignorants, incapables d'une concentration soutenue, insolents et apathiques [mais] accueillants, doux, généreux, et complaisants $»^{12}$. Ces plaintes sont aussi le témoignage d'un oubli des réalités administratives des pays "développés ", car d'aucuns admettent parfois que les procédures des organisations de coopération sont bien plus longues et pénibles que celles de l'administration laotienne.

8 L'Etat laotien, de son côté, change peu. L'économiste Chi Do Pham, depuis longtemps spécialiste du Laos, le reconnaît: "il faudrait un nouveau dispositif du même ordre de grandeur que les "nouveaux mécanismes économiques"13 [et la pensée nouvelle] pour relever le défi de l'intégration ${ }^{14}$ ». Les réformes prévues par le programme instauré en 1986 sont aujourd'hui pratiquement toutes en place, mais le Laos manque encore cruellement d'un environnement législatif à la hauteur des ambitions d'un Etat moderne, particulièrement concernant les droits de l'Homme et l'autorité même de la loi. La volonté de contrôle du gouvernement sur les acteurs de l'aide internationale, et particulièrement sur les organisations non gouvernementales, paraît avant tout être le reflet d'une volonté tenace de ne pas perdre le pouvoir - alors même que certains de ses membres hauts placés s'enrichissent par la corruption et l'argent détourné des projets. Le ralentissement des réformes économiques relève, pour Martin Stuart-Fox, de la même logique : "il existe une peur réelle de voir des réformes économiques radicales ouvrir la porte à des réformes politiques tout aussi radicales, jusqu'à ce que le Parti ne puisse plus empêcher l'avènement d'une démocratie multipartite. Et les dirigeants du Parti populaire révolutionnaire Lao savent très bien ce que le destin réserve aux partis communistes dans les pays nouvellement ouverts à la démocratie multipartite ${ }^{15}$ ". Mais si les progrès en matière d'ouverture économique ont été flagrants, on peut s'étonner du peu de progrès en matière de protection des droits de l'Homme et des libertés fondamentales. Une énergie et des sommes d'argent considérables ont été investies dans les réformes économiques (sous forme de prêts des institutions financières internationales, de dons de l'ONU et des coopérations bilatérales), alors que les projets et programmes pour la démocratie et les droits de l'Homme sont restés, jusqu'à très récemment, sur la touche ${ }^{16}$. Ce constat amène irrémédiablement à penser que les bailleurs institutionnels apportent plus d'importance à la rentabilité économique de leur aide qu'à l'émancipation des Laotiens. Dans ce contexte, les ONG ont clairement un rôle à jouer. Car si certaines d'entre-elles n'ont jamais prétendu lutter pour la défense des droits de l'Homme, d'autres en font (en partie, du moins) une mission constituante : CARE, MDM, MSF, Oxfam, etc. Mais au Laos, elles préfèrent se taire pour avoir accès aux populations dans le besoin. Elles préfèrent se taire pour pouvoir «travailler ». Ou continuer à avoir du travail. Car le Laos est un pays rêvé pour les ONG dites "de développement »: le gouvernement interdisant la création d'organisations non gouvernementales 
laotiennes, les ONG internationales ne peuvent, comme elles le font dans d'autres pays, «passer le flambeau » à un relais local. Elles peuvent donc justifier de leur présence indéfiniment.

9 Cependant, lorsqu'elles s'engagent à long terme, les ONG ont les moyens d'accumuler un savoir et une expérience qui peuvent être très judicieusement mis à profit. Mais ce n'est, hélas, pas le cas de toutes les organisations. Handicap International France, par exemple, avait mené des projets au Laos au milieu des années 1980 au début des années 1990. Les archives avaient été mises de côté par le responsable de mission, dans les bureaux de l'organisation à Bangkok. Quand la mission Laos a repris en 1997, ces archives n'ont pas été exploitées. Aujourd'hui, qui, chez Handicap International, sait que l'association était présente au Laos avant 1997 ? La rotation des postes, si elle est réduite chez certaines ONG, peut être aussi pesante que chez les bailleurs institutionnels, et anéantir tout aussi efficacement les relations de confiance tissées petit à petit avec les autorités locales.

La connaissance du «terrain » amène la compréhension des partenaires et le respect de leurs choix. Elle est fondamentale, particulièrement au Laos, si l'on ne veut pas faire de la " coopération pour le développement " une démarche unilatérale. Les ONG, que les croyances populaires imaginent favorisant la proximité et l'échange - plus que les acteurs gouvernementaux - semblent, en réalité, ne pas offrir de modèle à la hauteur de leur réputation et de leur démarche originelle.

\section{NOTES}

1.. AUSAID, Laos Australia Development Cooperation Program, 2004-2010, Commonwealth of Australia, 2005, Canberra, p. 9.

2.. Sogge D., Give and Take, What is the Matter with Foreign Aid?, Zed Books, Global issues series, London, 2002, p. 44-45.

3.. Médecins du Monde, Rapport moral, 1981.

4.. Pech T., Padis M. O., Les Multinationales du cœur. Les ONG, la politique et le marché, Paris, Seuil, La République des Idées, 2004, p. 79-80.

5.. Une personne en charge du recrutement dans une grande ONG, ibid.

6.. Brauman R., « Mission civilisatrice, ingérence humanitaire », in Le Monde Diplomatique, Paris, septembre 2005, p. 3.

7.. Olivier de Sardan J.-P., Anthropologie et développement : essai en socio-anthropologie du changement social, Paris, Karthala, 1995, p. 58.

8.. Ibid.

9.. Ibid.

10.. Brauman R., op. cit.

11.. Ibid.

12.. Taupin J., envoyé au Laos en 1887, cité dans Stuart-Fox M., Buddhist Kingdom, Marxist State : the Making of Modern Laos, Bangkok, White Lotus Press, 2002, p. 40-41.

13.. L'ouverture économique décidée par les dirigeants laotiens en 1986. 
14.. Pham C. D., The Lao Economy, Review of New Mechanism and the Challenge of Integration, Vientiane, The banking College of the Bank of the Lao PDR, 2004, p. 17.

15.. Stuart-Fox M., « Politics and Reform in the Lao PDR », in Political economy of development working paper $\mathrm{n}^{\circ} 1$, College of William and Mary, Williamsburg, USA, 2004, p. 26.

16.. La coopération suédoise semble être la seule à faire de la protection des droits de l'Homme une priorité de son programme.

INDEX

Thèmes : ONG

Index géographique : Asie du Sud-Est

Mots-clés : humanitaire 